\title{
COMPORTAMENTO DA PRESSÃO ARTERIAL EM HOMENS PRÉ-HIPERTENSOS PARTICIPANTES EM UM PROGRAMA REGULAR DE NATAÇÃO
}

\author{
BLOOD PRESSURE BEHAVIOUR IN PRE-HYPERTENSIVE MEN ENGAGED IN A REGULAR \\ SWIMMING PROGRAM \begin{abstract}
EN HOMBRES PRE-HIPERTENSOS
\end{abstract} \\ COMPORTAMIENTO DE LA PRESIÓN ARTERIAL DESPUÉS DE LA PRÁCTICA DE NATACIÓN
}

\begin{abstract}
Jairo Eleotério da Silva1,3 (Profissional de Educação Física)

Ana Maria Botelho Teixeira ${ }^{1}$ (Profissional de Bioquímica) Estélio Henrique Martin Dantas² (Profissional de Educação Física) Luis Manuel Pinto Lopes Rama ${ }^{1}$ (Profissional de Educação Física)
\end{abstract}

1. Faculdade de Ciências do Desporto e Educação Física Universidade de Coimbra (FCDEF-UC), Coimbra, Portugal. 2. Universidade do Estado do Rio de Janeiro (UNIRIO), Rio de Janeiro, RJ. 3. Universidade Castelo Branco (UCB), Rio de Janeiro, RJ, Brasil.

\section{Correspondência:}

Faculdade de Ciências do Desporto e Educação Física,

Estádio Universitário de Coimbra. Avenida Conímbriga - Pavilhão 3. 3040-248. Coimbra, Portugal. jairo.eleoterio@yahoo.com.br

\section{RESUMO}

Introdução: A natação tem sido recomendada como tipo de atividade física para a prevenção, tratamento e controle da hipertensão arterial. Objetivo: Analisar os efeitos da prática regular de natação sobre a pressão arterial de adultos pré-hipertensos. Métodos: A amostra foi composta por 36 homens pré-hipertensos divididos em dois grupos: Experimental - GE ( $n=24,40,60 \pm 9,36$ anos) e Controle - $G C$ ( $n=12,40,57 \pm 8,05$ anos). O GE realizou um programa regular de natação, com três sessões semanais de 45 min, durante 12 semanas, enquanto o GC não alterou seus hábitos alimentares e permaneceu sem praticar atividade física no período. O procedimento estatístico utilizado para verificar as diferenças entre as médias antes e depois de 12 semanas do programa regular de natação foi o teste-t de Student. O estudo admitiu o nível de $p<0.05$ para a significância estatística. Resultados: $O$ grupo GE apresentou diferenças estatisticamente significativas nas variáveis analisadas quando comparadas com o GC, tanto na pressão arterial sistólica, PAS $(\Delta=-5,89 \mathrm{mmHg}, p=0,00)$, como na pressão arterial diastólica, $\operatorname{PAD}(\Delta=-5,15 \mathrm{mmHg}, \mathrm{p}=0,00)$. Conclusão: Os resultados mostram que um programa regular de natação provoca reduções significativas na pressão arterial em repouso em homens pré-hipertensos.

Palavras-chave: atividade física, exercício, natação, hipertensão, pré-hipertensão, pressão sanguínea.

\section{ABSTRACT}

Introduction: Swimming as a modality of physical activity that has been recommended for preventing, controlling and treating arterial hypertension. Objective: To verify the persistent effects of a regular swimming program on pre-hypertensive adults on arterial pressure levels. Methods: The sample was constituted of 36 pre-hypertense individuals who were divided into two groups: experimental - GE ( $n=24,40.60 \pm 9.36$ years old ) and control - GC ( $n=12,40.57 \pm 8.05$ years old. GE individuals took part in a regular swimming programme consisting of three forty-five training sessions weekly (ST) for 12 weeks, whereas GC individuals were instructed not to alter their nutritional habits and did not practice any physical activity during the experiment. The Student $t$-test was used as statistical procedure to analyze the differences between the average indexes recorded before and after the 12-weeks regular swimming program. The study admitted a level statistical significance at $p$ $<0.05$ for. Results: The results led to the conclusion that experimental group presented a statistically significant difference for the analyzed variables when compared to Control group. There was an important reduction of systolic blood pressure, SBP $(\Delta=-5,89 \mathrm{mmHg}, p=0,00)$, and also of diastolic blood pressure, $\mathrm{DBP}(\Delta=-5,15 \mathrm{mmHg}, p=0,00)$. Conclusion: The results showed that a regular swimming program leads to a significant decrease in resting blood pressure values of pre-hypertensive men.

Keywords: motor activity, exercise, swimming, hypertension, prehypertension, blood pressure.

\section{RESUMEN}

Introducción: La natación ha sido recomendada como un tipo de actividad física para la prevención, tratamiento y control la de hipertensión arterial. Objetivo: Analizar los efectos de la práctica regular de natación sobre la presión arterial de adultos prehipertensos. Métodos: La muestra se ha compuesto por 36 hombres con prehipertensión delineados en dos grupos: experimental - GE ( $n=24,40,60 \pm 9,36$ años) y Control - GC ( $n=12,40,57 \pm 8,05$ años). El GE realizó un programa regular de natación, con tres sesiones semanales, duración de 45 minutos, durante 12 semanas, mientras que el GC no cambió los hábitos alimenticios y permaneció sin realizar actividad física en el período. El procedimiento estadístico utilizado para averiguar las diferencias entre los promedios antes y después de 12 semanas del programa regular de natación fue el test t de Student. El estudio admitió el nivel de $p<0.05$ para la significancia estadística. Resultados: El grupo GE presentó diferencias estadísticamente significativas en las variables analizadas, cuando comparadas con el GC, tanto en la presión arterial sistólica, PAS $(\Delta=-5,89 \mathrm{mmHg}, P$ $=0,00)$ como en la presión arterial diastólica, $P A D(\Delta=-5,15 \mathrm{mmHg}, p=0,00)$. Conclusión: Los resultados muestran que un programa regular de natación provoca reducciones significativas en la presión arterial en reposo en hombres prehipertensos.

Palabras clave: actividad física, ejercicio, natación, hipertensión, pre-hipertensión, presión sanguínea. 


\section{INTRODUÇÃO}

Os programas de atividade física sistematizados representam a abordagem não farmacológica inicial para a prevenção e tratamento da Hipertensão Arterial (HTA) ${ }^{1-3}$. A HTA pode ser conceituada como uma condição clínica multifatorial, caracterizada por níveis elevados e sustentados de pressão arterial (PA) e sua presença pode causar lesões em órgãos vitais, tais como coração, cérebro e rins ${ }^{1,4}$. Os limites que definem HTA considera valores de Pressão Arterial Sistólica (PAS) $\geq 140 \mathrm{mmHg}$ e/ou de Pressão Arterial Diastólica (PAD) $\geq 90 \mathrm{mmHg}$. A prevalência da HTA tem sido um dos maiores problemas de saúde em todo o mundo, podendo afetar aproximadamente um bilhão de pessoas. Somente nos Estados Unidos estima-se uma prevalência entre $24 \%$ a $29 \%$ de adultos economicamente ativos $2,3,5,6$. No Brasil estes dados são muito semelhantes, pois existe uma estimativa de 22 a $44 \%$ de hipertensos ${ }^{1,4,7}$.

Buscando minimizar a possibilidade crescente da HTA em todo o mundo, houve uma alteração importante na classificação tradicional sobre HTA, aparecendo o termo pré-hipertensão (PH), cujos níveis de PA situam-se entre 120 e $139 \mathrm{mmHg}$ para a PAS e/ou entre 80 e $89 \mathrm{mmHg}$ para a PAD. O termo PH foi descrito em 2003 pela Diretriz Americana de Hipertensão Arterial ${ }^{5}$, que passou a enfatizar medidas preventivas mais rigorosas para o comportamento da PA, uma vez que indivíduos, na faixa etária de 40 a 49 anos, classificados como pré-hipertensos, possuem $80 \%$ de probabilidade de apresentar hipertensão nos dez anos subsequentes ${ }^{1,2,8}$.

Classicamente algumas mudanças nos hábitos alimentares e a adoção de um estilo de vida ativo, com a inclusão do exercício ou atividade física regular representam requisitos essenciais para a prevenção e tratamento da hipertensão. Embora haja um largo espectro de diferentes tipos de atividades físicas recomendadas como forma de prevenir e tratar a HTA, existem poucas pesquisas que demonstram ser

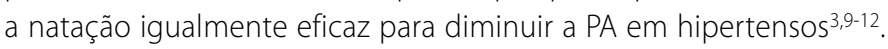
Segundo Fagard ${ }^{9}$ apenas $3 \%$ das pesquisas relacionadas utilizam a natação como tratamento da hipertensão em seres humanos. O fato de se utilizar outras modalidades de exercício e/ou atividade física em detrimento da natação pode ser explicado pelos seguintes fatores: Em primeiro lugar, deve-se considerar as dificuldades iniciais da realização de medidas fisiológicas na água e segundo, a natação requer certas habilidades e técnicas para se alcançar determinados níveis de intensidade do exercício, quando comparado a outras modalidades $^{13}$. O objetivo deste estudo foi analisar os efeitos de um programa de prática regular de natação sobre a pressão arterial de homens pré-hipertensos.

\section{MATERIAL E MÉTODOS}

Foram selecionados homens pré-hipertensos, recrutados através de anúncios em rádio e jornais e que apresentaram um atestado médico confirmando sua condição de participar do experimento. Nenhum deles apresentou qualquer sintoma de doença coronária, infarto do miocárdio, insuficiência cardíaca, diabetes, doenças neuromusculares ou outra similar que pudesse impedi-los de praticar natação. Antes da intervenção foram dadas explanações verbais e escritas do procedimento experimental, benefícios e possíveis riscos para os participantes. Todos os sujeitos assinaram o Termo de Consentimento Livre e Esclarecido (TCLE) concordando em participar do experimento.

O presente estudo atende às normas para a realização de pesquisas com seres humanos, conforme a Resolução n 466, de 12 de dezembro de 2012, do Conselho Nacional de Saúde, e foi aprovado no Comitê de Ética em Pesquisa da UCB/ RJ, sob no 0037/2007.
Um total de 36 voluntários do sexo masculino, pré-hipertensos, foram selecionados aleatoriamente para este estudo e alocados em dois grupos: Experimental - GE ( $n=24,40,60 \pm 9,36$ anos) e Controle - GC ( $n=12,40,57 \pm 8,05$ anos. Os sujeitos do grupo experimental participaram de um programa regular de natação, enquanto o grupo controle não praticou nenhuma atividade física no período.

\section{Protocolo de aferições e medidas}

Os indivíduos do grupo experimental participaram de um programa regular de natação, três vezes semanais, com duração de 40 a 45 min cada sessão, durante um período de 12 semanas. Cada sessão foi dividida da seguinte forma: 5 min de alongamento e aquecimento, 30 a 35 min de natação e, ao final, 5 min de relaxamento e volta à calma.

A massa corporal e a estatura foram medidas por meio de uma balança de precisão Filizola ${ }^{\circledR}$ (Brasil), com os indivíduos trajando roupa esportiva. A Pressão Arterial foi mensurada segundo as recomendações do diagnóstico de hipertensão do ACSM, sendo aferida por aparelho oscilométrico (Omrom, modelo HEM-741C, Japão). Este equipamento recebeu a validação da British Hypertension Society (BHS) e da Association for Advanced in Medical Instruments (AAMI) ${ }^{14}$. A intensidade do exercício foi controlada através da utilização de um monitor de frequência cardíaca (FC) da marca Polar ${ }^{\circledR}$ (modelo F5, Finlândia), respeitando um período de repouso entre cinco e 10 min pré e pós-exercício. As características físicas e parâmetros hemodinâmicos dos sujeitos são mostrados na tabela 1.

Para o programa de natação não era necessário que os sujeitos soubessem nadar. As sessões iniciais tiveram uma intensidade em torno de $40 \%$ a $50 \%$ da frequência cardíaca máxima $\left(F C_{\max }\right.$ ) predita através da equação de Tanaka et al. ${ }^{15}$ 2001. Cerca de 20\% do trabalho aquático foi constituído de movimentos de pernada, utilizando-se uma prancha de natação. A temperatura da água da piscina se manteve em torno de $27-29^{\circ} \mathrm{C}$ durante as doze semanas da intervenção.

Tabela 1. Características físicas e hemodinâmicas dos sujeitos.

\begin{tabular}{|c|c|c|c|}
\hline & $\mathrm{GE}(n=24)$ & $\mathrm{GC}(\mathrm{n}=12)$ & Valor de $p$ \\
\hline \multicolumn{4}{|c|}{ Características físicas } \\
\hline Idade (anos) & $40.60 \pm 9.36$ & $40.57 \pm 8.05$ & 0.620 \\
\hline \multicolumn{4}{|l|}{ MC (kg) } \\
\hline Pré & $59.2 \pm 12$ & $76.7 \pm 16.3$ & 0.040 \\
\hline Pós-exercício & $59.3 \pm 12.4 \#$ & $76.4 \pm 16.3 \#$ & 0.060 \\
\hline Estatura $(\mathrm{cm})$ & $149 \pm 9$ & $156.5 \pm 7.4$ & 0.430 \\
\hline \multicolumn{4}{|l|}{$\mathrm{IMC}\left(\mathrm{kg} / \mathrm{m}^{2}\right)$} \\
\hline Pré & $23.9 \pm 3.4$ & $26.4 \pm 4.1$ & 0.493 \\
\hline Pós-exercício & $23.7 \pm 3.3 \#$ & $26.3 \pm 3.9 \#$ & 0.471 \\
\hline \multicolumn{4}{|c|}{ Dados hemodinâmicos } \\
\hline \multicolumn{4}{|l|}{ PAS (mmHg) } \\
\hline Pré & $136.49 \pm 3.82$ & $138.49 \pm 3.62$ & 0.042 \\
\hline Pós-exercício & $130.60 \pm 3.66^{*}$ & $138.60 \pm 3.86$ & 0.053 \\
\hline \multicolumn{4}{|l|}{ PAD $(\mathrm{mmHg})$} \\
\hline Pré & $87.96 \pm 3.63$ & $89.96 \pm 3.93$ & 0.030 \\
\hline Pós-exercício & $82.81 \pm 2.75^{*}$ & $88.82 \pm 2.45$ & 0.023 \\
\hline
\end{tabular}

Valores:média \pm DP; MC- massa corporal; IMC índice de massa corporal; PAS - pressão arterial sistólica; PAD pressão arterial diastólica. Diferença significativa (*) e Diferença Não-significativa (\#) em relação ao início do programa $(p<0,05)$. 


\section{Análise estatística}

Os dados coletados foram digitados no programa Microsoft Excel e analisados no programa SPSS (versão 17.0, Chicago, IL, USA). O teste Shapiro-Wilk foi usado para verificar se os dados numéricos apresentaram distribuição normal. Para a análise comparativa foram utilizados os testes $t$ Student. O nível de significância adotado foi de $p<0,05$.

\section{RESULTADOS}

Em relação as variáveis massa corporal (MC) e índice de massa corporal (IMC), pode-se notar que não existem diferenças significativas em ambos os grupos, para valores iniciais e ao final do programa de natação (tabela 1).

No que se refere as pressões arteriais tanto sistólicas como diastólicas, observam-se diferenças significativas apenas no GE (redução de 5,89 $\mathrm{mmHg}$ para PAS e 5,15 para a PAD) (figuras 1 e 2).

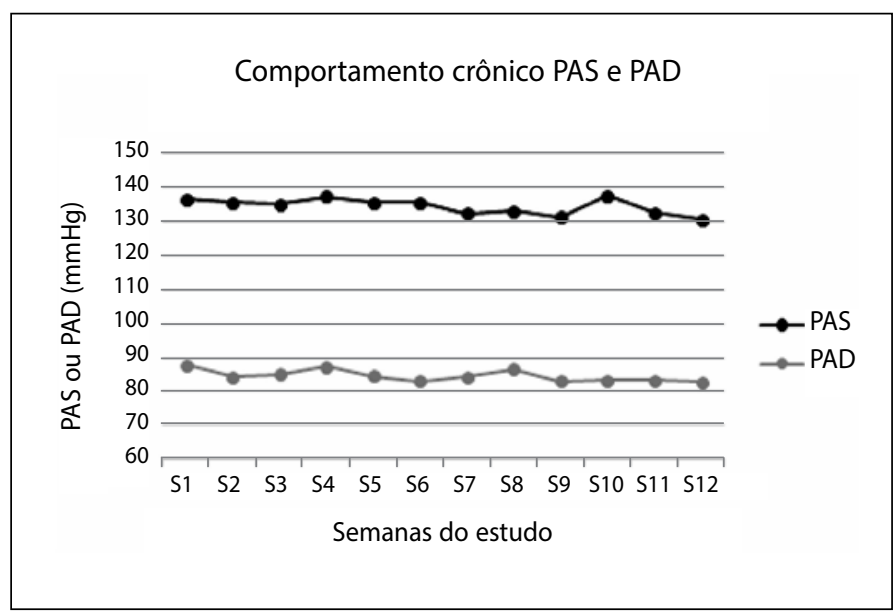

Figura 1. Comportamento das PAS e PAD do GE durante 12 semanas de natação.

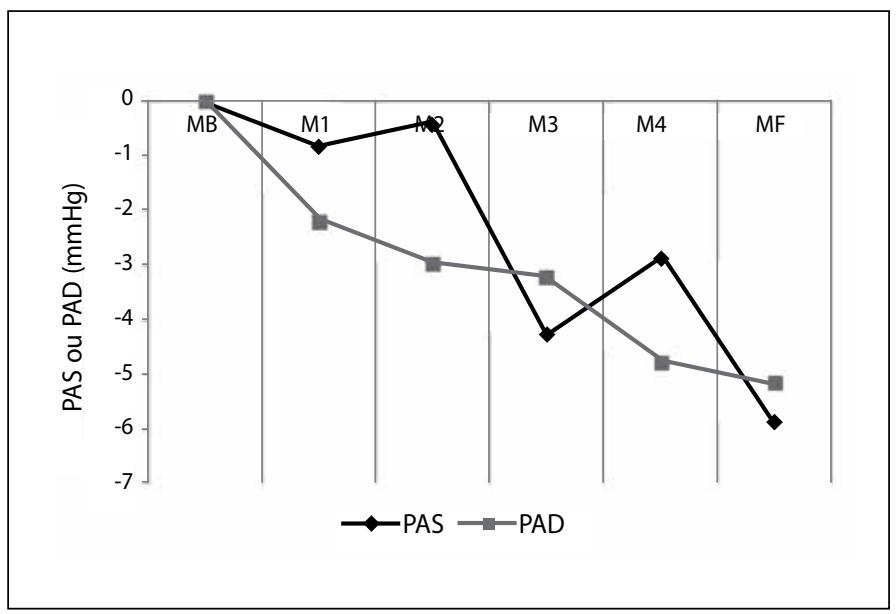

Figura 2. Delta da variação da PA sistólica (PAS) e diastólica (PAD) do GE, após o programa de 12 semanas de natação, durante os momentos MB-momento basal, M1 a M4 e MF- momento final. PA - pressão arterial.

\section{DISCUSSÃO}

Em nosso estudo encontramos redução significativa tanto na PA sistólica como na diastólica de homens pré-hipertensos. Ressalta-se aqui o importante efeito hipotensor da natação que pode resultar em proteção contra eventos cardiovasculares como proposto por Whelton et al. ${ }^{16}$ ou seja, uma redução de apenas $3 \mathrm{mmHg}$ para a PAS, pode significar diminuição de 5-9\% e 8-14\% para risco cardiovascular e infarto do miocárdio respectivamente. A magnitude de redução de nossa pesquisa para as PA sistólica e diastólica representa quase duas vezes o que foi proposto por Whelton et al. ${ }^{16}$ (2002). A natação caracteriza-se por ser um tipo de atividade física que envolve grandes grupos musculares, é uma atividade ritmada, dinâmica e aeróbica ${ }^{13}$, e apresenta uma série de vantagens: (1) realizada no plano horizontal; (2) está sujeita à ação da pressão hidrostática e (3) tem como efeito o aumento do retorno venoso e do volume sistólico ${ }^{17}$. Embora sua ampla recomendação por diversas organizações nacionais e internacionais relacionadas a saúde ${ }^{1,2,8}$ para prevenção, controle e tratamento da HTA, existem poucas pesquisas que relacionam hipertensão e natação em seres humanos ${ }^{3,13,18}$. Além disso, a literatura reporta resultados conflitantes em relação aos efeitos da natação na PA em indivíduos hipertensos ${ }^{11,19}$

Nossos resultados corroboram estudos anteriores ${ }^{11,20-22}$ onde também foram encontradas diferenças significativas para algumas variáveis relacionadas também em nossa pesquisa. Entretanto Tanaka et al. ${ }^{11}$ e Nualnim et al. ${ }^{21}$ encontraram diferenças significativas para a variável pressão arterial sistólica, enquanto Chen et al. ${ }^{22}$ encontraram normalização mais do que redução na PA, após um ano de intervenção. Enfatizamos que a magnitude da redução mostra-se ainda mais importante por se tratar de indivíduos pré-hipertensos, pois nestes estudos supracitados as reduções referem-se a indivíduos hipertensos, uma vez que se sabe que as reduções mostram-se maiores em hipertensos, ou seja, em pressões arteriais mais altas.

Vários são os mecanismos que explicam a hipotensão pós-exercício (HPE) resultante de uma sessão (efeito agudo) ou de várias sessões de exercício (efeito crônico) sobre a PA de indivíduos normotensos, pré-hipertensos e hipertensos. Nesta linha de raciocínio, diversos estudos afirmam que a causa da HPE pode ser explicada pelos seguintes fatores: (a) diminuição da resistência vascular periférica (RVP), b) diminuição do débito cardíaco (DC) e redução da atividade nervosa simpática e/ou d) uma combinação dos fatores anteriores ${ }^{23-27}$.

Por fim os resultados deste estudo sugerem que reduções na PA podem acontecer independente de alterações da massa corporal e índice de massa corporal, conforme descrito por Blumenthal et al. ${ }^{28}$ Merece ainda relevância pelo fato do procedimento experimental clarificar a compreensão da associação entre a HPE e a prática da natação. No entanto não podem ser ignorados que fatores nutricionais poderão ter influenciado os resultados por nós obtidos. O fato de não ter controlado o regime nutricional dos sujeitos pode ser considerado uma limitação do presente estudo.

\section{CONCLUSÃO}

Ao final do programa de natação, houve diferenças significativas nas variáveis analisadas quando comparadas ao grupo controle. Encontramos uma redução estatisticamente significativa para pressão sistólica (PAS) e para pressão diastólica (PAD).Os resultados nos mostram que a natação regular provoca diminuições significativas nas pressões sistólica e diastólica em homens pré-hipertensos.

\section{AGRADECIMENTOS}

O autor Jairo Eleotério da Silva, Bolsista de Doutoramento Pleno no Exterior (CNPq) Universidade de Coimbra, Coimbra, Portugal.

Todos os autores declararam não haver qualquer potencial conflito de interesses referente a este artigo. 


\section{REFERÊNCIAS}

1. Sociedade Brasileira de Hipertensão, Sociedade Brasileira de Cardiologia e Sociedade Brasileira de Nefrologia. IV Diretrizes Brasileiras de Hipertensão Arterial. Arq Bras Cardiol. 2004;82(Supl 4):1-14

2. Bath P, Chalmers J, Powers W, Beilin L, Davis S, Lenfant C, et al. International Society of Hypertension (ISH): statement on the management of blood pressure in acute stroke. J Hypertens. 2003;21(4):665-72.

3. Pescatello LS, Franklin BA, Fagard R, Farquhar WB, Kelley GA, Ray CA; American College of Sports Medicine. American College of Sports Medicine position stand. Exercise and hypertension. Med Sci Sports Exerc. 2004;36(3):533-53.

4. VI Diretrizes Brasileiras de Hipertensão. Arq Bras Cardiol 2010; 95(1 Supl.1):1-51.

5. Chobanian AV, Bakris GL, Black HR, Cushman WC, Green LA, Izzo Jr JL, et al. The seventh report of the joint national committee on prevention, detection, evaluation, and treatment of high blood pressure: the JNC 7 report. Jama. 2003;289(19):2560-71.

6. Wolf-Maier K, Cooper RS, Kramer H, Banegas JR, Giampaoli S, Joffres MR, et al. Hypertension treatment and control in five European countries, Canada, and the United States. Hypertension. 2004:43(1):10-7.

7. Sociedade Brasileira de Cardiologia-SBC; Sociedade Brasileira de Hipertensão-SBH; Sociedade Brasileira de Nefrologia-SBN. V Brazilian Guidelines in Arterial Hypertension. Arq Bras Cardiol. 2007;89(3):e24-79.

8. Mancia G, De Backer G, Dominiczak A, Cifkova R, Fagard R, Germano G, et al. 2007 Guidelines for the management of arterial hypertension: The Task Force for the Management of Arterial Hypertension of the European Society of Hypertension (ESH) and of the European Society of Cardiology (ESC). Eur Heart J. 2007;28(12):1462-536.

9. Fagard RH. Exercise characteristics and the blood pressure response to dynamic physical training. Med Sci Sports Exerc. 2001;33(6 Suppl):S484-92;

10. Silva J, Geraldes A, Natali A, Pereira J, Vale R, Dantas E. Acute effects of swimming on the arterial pressure of hypertensive adults. Maced J Med Sci. 2009;2(4):330-4.

11. Tanaka H, Bassett DR Jr, Howley ET, Thompson DL, Ashraf M, Rawson FL. Swimming training lowers the resting blood pressure in individuals with hypertension. J Hypertens. 1997;15(6):651-7.

12. Anunciação $P G$, Polito MD. Hipotensão pós-exercício em indivíduos hipertensos: uma revisão. Arq Bras Cardiol. 2011;96(5):425-6.

13. Tanaka H. Swimming exercise: impact of aquatic exercise on cardiovascular health. Sports Med 2009;39(5):377-87.

14. O'Brien E, Pickering T, Asmar R, Myers M, Parati G, Staessen J, et al. Pressure Monitoring of the European Society of Hypertension. Working Group on Blood Pressure Monitoring of the European Society of
Hypertension International Protocol for validation of blood pressure measuring devices in adults. Blood Press Monit. 2002;7(1):3-17.

15. Tanaka H, Monahan KD, Seals DR. Age-predicted maximal heart rate revisited. J Am Coll Cardiol. 2001;37(1):153-6

16. Whelton SP, Chin A, Xin X, He J. Effect of aerobic exercise on blood pressure: a meta-analysis of randomized, controlled trials. Ann Intern Med. 2002;136(7):493-503.

17. Esteves LMZ, Simões HG, Oliveira SML, Cunha VNC, Coelho JMO, Botelho Neto W, et al. Respostas cardiovasculares pós-exercício de natação. Rev Bras Med Esporte. 2010;16(6):418-21.

18. Fagard RH. Physical activity, physical fitness and the incidence of hypertension. J Hypertens. 2005;23(2):265-7.

19. Cox KL, Burke V, Beilin LJ, Grove JR, Blanksby BA, Puddey IB. Blood pressure rise with swimming versus walking in older women: the Sedentary Women Exercise Adherence Trial 2 (SWEAT 2). J Hypertens. 2006;24(2):307-14.

20. da Silva JE, Pereira JS, Natali AJ, de Souza Vale RG, Dantas EHM. Efectos crónicos de un programa regula de natación. Sobre la tensión arterial de adultos hipertensos. Rev Int Cienc Deporte. 2006;2(4):15-24

21. Nualnim N, Parkhurst K, Dhindsa M, Tarumi T, Vavrek J, Tanaka H. Effects of swimming training on blood pressure and vascular function in adults $>50$ years of age. Am J Cardiol. 2012;109(7):1005-10.

22. Chen HH, Chen YL, Huang CY, Lee SD, Chen SC, Kuo CH. Effects of one-year swimming training on blood pressure and insulin sensitivity in mild hypertensive young patients. Chin J Physiol. 2010;53(3):185-9.

23. Blanchard BE, Tsongalis GJ, Guidry MA, LaBelle LA, Poulin M, Taylor AL, et al. RAAS polymorphisms alter the acute blood pressure response to aerobic exercise among men with hypertension. Eur Appl Physiol. 2006;97(1):26-33.

24. Hagberg JM, Park JJ, Brown MD. The role of exercise training in the treatment of hypertension: an update. Sports Med. 2000;30(3):193-206

25. Quinn TJ. Twenty-four hour, ambulatory blood pressure responses following acute exercise: impact of exercise intensity. J Hum Hypertens. 2000;14(9):547-53.

26. Rogers MW, Probst MM, Gruber JJ, Berger R, Boone JB Jr. Differential effects of exercise training intensity on blood pressure and cardiovascular responses to stress in borderline hypertensive humans. Hypertens. 1996;14(11):1369-75.

27. Brandão Rondon MU, Alves MJ, Braga AM, Teixeira OT, Barretto AC, Krieger EM, et al. Postexercise blood pressure reduction in elderly hypertensive patients. J Am Coll Cardiol. 2002;39(4):676-82

28. Blumenthal JA, Sherwood A, Gullette EC, Babyak M, Waugh R, Georgiades A, et al. Exercise and weigh loss reduce blood pressure in men and women with mild hypertension: effects on cardiovascular, metabolic, and hemodynamic functioning. Arch Intern Med. 2000;160(13):1947-58. 